\title{
Short communication: Effect of inhibition of fatty acid synthase on triglyceride accumulation and effect on lipid metabolism genes in goat mammary epithelial cells
}

\author{
J. J. Zhu, ${ }^{,}$J. Luo, ${ }^{* 1}$ Y. T. Sun, ${ }^{*}$ H. B. Shi, ${ }^{,}$J. Li, ${ }^{*}$ M. Wu, ${ }^{*}$ K. Yu, ${ }^{*}$ A. B. Haile, ${ }^{*}$ and J. J. Loor† \\ *Shaanxi Key Laboratory of Molecular Biology for Agriculture, College of Animal Science and Technology, Northwest A\&F University, Yangling, \\ Shaanxi, P. R. China 712100 \\ †Mammalian NutriPhysioGenomics, Department of Animal Sciences and Division of Nutritional Sciences, University of Illinois, Urbana 61801
}

\begin{abstract}
The role of fatty acid synthase (FASN) on de novo fatty acid synthesis has been well established. In monogastrics, unlike acetyl-coenzyme A carboxylase, FASN is primarily controlled at the transcriptional level. However, no data exist on ruminant mammary cells evaluating effects of FASN knockdown on mRNA expression of lipogenic genes. Inhibition of FASN in mammary cells by C75-mediated interference, a synthetic inhibitor of FASN activity, and short hairpin RNA-mediated interference markedly reduced cellular triglyceride content at least in part by decreasing the expression of genes related to triglyceride synthesis (GPAT, AGPAT6, and DGAT2) and enhancing the expression of lipolysis-related genes (ATGL and $H S L)$. Consistent with the markedly lower expression of genes related to lipid droplet formation and secretion (TIP 47, $A D F P, B T N 1 A 1$, and $X D H)$, cellular lipid droplets also were reduced sharply after incubation with $\mathrm{C} 75$ or adenovirus-short-hairpin-RNA. The results underscored the essential role of FASN in the overall process of milk-fat formation in goat mammary epithelial cells. Key words: fatty acid synthase, milk fat, goat mammary epithelial cell
\end{abstract}

\section{Short Communication}

Milk fat is an important component of the nutritional quality of goat dairy products (Bernard et al., 2005). It is composed of $98 \%$ triglyceride (TAG; Dils, 1986), which is present in unique membrane-bound structures referred to as milk lipid globules (Hansen et al., 1984; De Schrijver et al., 2003). Milk lipids originate from cellular lipid droplets; these are secreted from mammary epithelial cells (Chong et al., 2011). Milk fat is an essential contributor to many of the physical properties,

Received April 2, 2014.

Accepted January 12, 2015.

${ }^{1}$ Corresponding author: luojun@nwsuaf.edu.cn manufacturing qualities, and organoleptic characteristics of dairy products (Harvatine et al., 2009). Some FA found in milk, such as oleic and linolenic acid, also have been shown to be beneficial to human health (Massaro et al., 1999).

The FA esterified in TAG have 2 main origins, de novo synthesis in the mammary gland or extraction from the arterial blood (Bernard et al., 2005). In ruminants, FA synthase (FASN) catalyzes successive condensation of malonyl-CoA and acetyl-CoA to form FA, including palmitate, myristate, laureate, and even shorter-chain FA (Menendez et al., 2009; Currie et al., 2013). Lower activity of FASN in mice decreased lipid droplet size and stimulated efflux of FFA and glycerol from adipocytes (Kurokawa et al., 2010). Despite the important roles of FASN in regulating the expression of genes related to lipid metabolism in goat mammary epithelial cells (GMEC; Wang, 2009), the exact mechanism of the involvement of FASN in the regulation of TAG synthesis and milk fat-droplets formation is largely unknown.

The hypothesis for this study was that downregulation of FASN mRNA expression would cause alterations in the expression of other lipogenic genes and cause changes in TAG synthesis. The specific objective was to downregulate the expression of FASN using short hairpin RNA (shRNA)-mediated RNA interference and C75, a specific inhibitor of FASN activity, and measure the expression of lipogenic genes and accumulation of lipid droplets.

To detect how inhibition of FASN affects TAG synthesis, we used C75 or shRNA for knocking down FASN. Inhibitor C75 is a widely used inhibitor of mammalian FASN and mainly targets $\beta$-ketoacyl synthase (Rendina and Cheng, 2005). In diet-induced obese mice, the inhibition of FASN in adipocytes by $\mathrm{C} 75$ suppressed de novo FA synthesis and increased energy utilization and FA oxidation, despite a high level of malonyl-CoA (Thupari et al., 2002). Such responses were due to a stimulation of carnitine palmitoyltransferase-1 (CPT-1) activity in adipose tissue and fatty liver. 
In the present study, different concentrations of C75 (0, 1, 5, 7, and $10 \mathrm{ng} / \mu \mathrm{L}$; Sigma, St. Louis, MO) were used for GMEC incubation for $48 \mathrm{~h}$. Goat mammary epithelial cells were cultured as described in the Supplementary Material (http://dx.doi.org/10.3168/ jds.2014-8202). The results of activity determination revealed that $10 \mathrm{ng} / \mu \mathrm{L}$ of $\mathrm{C} 75$ was most effective in inhibiting FASN activity without a morphological change in GMEC (Zhu et al., 2014; data not shown). For the following study, $10 \mathrm{ng}$ of $\mathrm{C} 75 / \mu \mathrm{L}$ was used for GMEC treatment. Goat mammary epithelial cells were seeded in $4.5-\mathrm{cm}^{2}$ cell-culture clusters (NUNC, Shanghai, China) for $24 \mathrm{~h}$ (approximately $90 \%$ confluent) before treatment with $10 \mu \mathrm{g} / \mathrm{mL}$ of C75 (dissolved in dimethyl sulfoxide; Sigma) for 48 h in Dulbecco's modified Eagle medium/nutrient mixture F-12 complete medium. Using the same method, we also treated GMEC in $10-\mathrm{cm}^{2}$ clusters (NUNC) with C75 to evaluate TAG synthesis (Applygen, Beijing, China) and lipid-droplet formation (oil red O staining; Sigma) as explained in detail in the Supplementary Material (http://dx.doi.org/10.3168/ jds.2014-8202). Prior to experiments, cells were washed twice with PBS and once with serum-free Dulbecco's modified Eagle medium/nutrient mixture F-12 without antibiotics, and $1.5 \mu \mathrm{g} / \mathrm{mL}$ of prolactin (CAS: 9002-624, Sigma) was supplemented to the culture medium of GMEC (Peterson et al., 2004; Kadegowda et al., 2009).

Cellular total RNA was extracted and purified with the Cell RNA Extraction Kit with DNase (Tiangen, Beijing, China). First-strand complementary DNA was synthesized from $0.5 \mu \mathrm{g}$ of purified total RNA using the PrimeScript RT kit (for perfect real time; Takara, Otsu, Japan) according to the manufacturer's instructions. The SYBR Premix Ex Taq II (Takara) was used for measuring mRNA expression by quantitative real-time (RT)-PCR according to the manufacturer's manual on CFX96 Real-Time PCR Detection System (Bio-Rad, Hercules, CA). Quantitative RT-PCR from each sample was performed in triplicate. Glyceraldehyde phosphate dehydrogenase (GAPDH; Yonezawa et al., 2008, 2009), ubiquitously expressed transcript (UXT), and mitochondrial ribosomal protein L39 (MRPL39; Kadegowda et al., 2009) were used as internal control genes. The FASN protein expression was determined according to our previous experiment (Zhu et al., 2014). The determination of FASN mRNA abundance in the treatments is reported in Supplemental Figure S1 (http://dx.doi.org/10.3168/ jds.2014-8202). The primer sequences are shown in Supplemental Table S1 (http://dx.doi.org/10.3168/ jds.2014-8202). Software SPSS 19.0 was used for the statistical analysis as described in the Supplementary Material (http://dx.doi.org/10.3168/jds.2014-8202).

In the present study, compared with the control group (dimethyl sulfoxide), the inhibition of FASN by
C75 significantly decreased mRNA expression of genes associated with TAG synthesis, including GPAM by $32 \%, A G P A T 6$ by $25 \%$, and DGAT2 by $45 \%$ (except $D G A T 1$ ), and increased the expression of the lipolytic genes hormone-sensitive lipase $(H S L)$ by 1.69 -fold and adipose triglyceride lipase $(A T G L)$ by 1.83 -fold. In contrast, the expression of LPIN1 had no obvious change. An unexpected inhibitory effect was observed on the expression of genes related to lipid-droplet formation and secretion, including tail-interacting protein 47 gene $\left(T I P 4^{7}\right)$, adipose differentiation-related protein $(A D F P)$, xanthine oxidoreductase $(X D H)$, and butyrophilin 1A1 (BTN1A1), which decreased by 32, 47, 70, and $69 \%$, respectively (Figure $1 \mathrm{~A}$ ).

In the present study, after the treatment of GMEC with $\mathrm{C} 75$, the cellular TAG content decreased $(P<$ 0.05 ) by $41 \%$. Consistent with that reduction, the accumulation of lipid droplet decreased $(P<0.05)$ by $8 \%$ with $\mathrm{C} 75$ compared with the dimethyl sulfoxide group (Figure 2A).

Similar to the effect observed with C75 treatment, after infection with Ad-shRNA, shRNA targeting FASN, the mRNA expression of FASN decreased $(P<0.05)$ by $47 \%$ and the protein expression by $46 \%$ (Figure $\mathrm{S} 1$; http://dx.doi.org/10.3168/jds.2014-8202). Similar to C75, the expression of GPAM, AGPAT6, and DGAT2 was decreased by 60,21 , and $60 \%$ in response to AdshRNA compared with the control group (Ad-NC; $P<$ 0.05). However, opposite to the decrease in the expression of genes related to lipid synthesis, the expression of $H S L$ increased $(P<0.05)$ by 2.87 -fold. Similar to the results with $\mathrm{C} 75$ treatment, the reduction in FASN gene expression was associated with lower $(P<0.05)$ expression of TIP 47 (by 59\%), ADFP (by $51 \%$ ), $X D H$ (by $60 \%$ ) and BTN1A1 (by 65\%). The expression of $L P I N 1$ and DGAT1 remained unaffected $(P>0.05$; Figure 1B). The abundance of TAG after infection with adenovirus-shRNA (Ad-shRNA) decreased by $55 \%(P$ $<0.05)$. This decrease was validated by the reduced accumulation of lipid droplets in GMEC $(-23 \% ; P<$ 0.05; Figure 2B).

Undoubtedly the role of FASN on de novo FA synthesis has been well-established in human (Chirala et al., 2001), bovine (Morris et al., 2007), Caenorhabditis elegans (Olsen, 2011), and mice (Suburu et al., 2014). The positive relationship between the expression of FASN and TAG synthesis and accumulation also has been well studied. In human prostate-cancer cells, RNA interference-mediated silencing of FASN decreases accumulation of neutral lipids (De Schrijver et al., 2003). In mice, a soy $\beta$-conglycinin diet reduced serum TAG levels and suppressed FASN (Moriyama et al., 2004). However, these studies could not shed light on the physiological effect in the mammary gland of ruminants. Although 
A

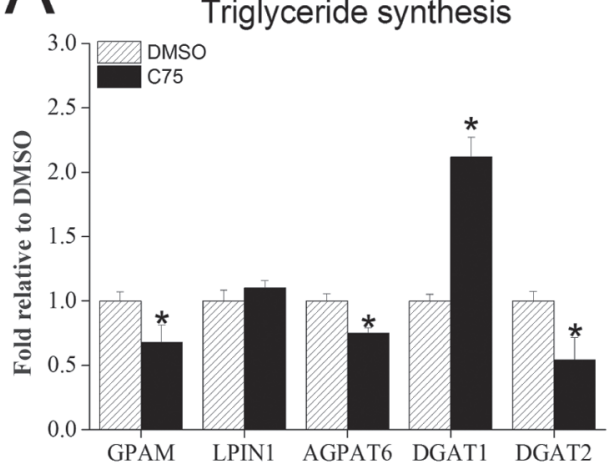

B

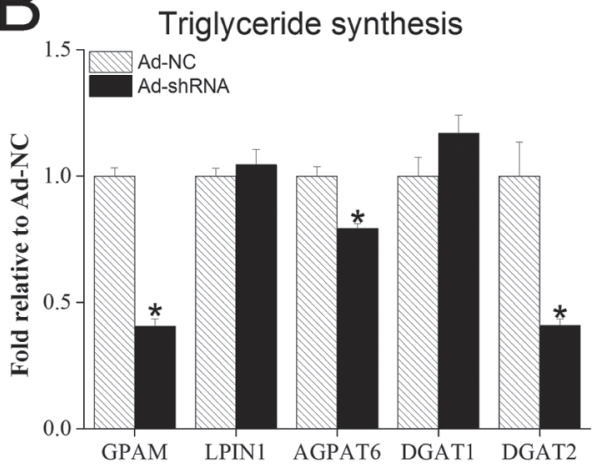

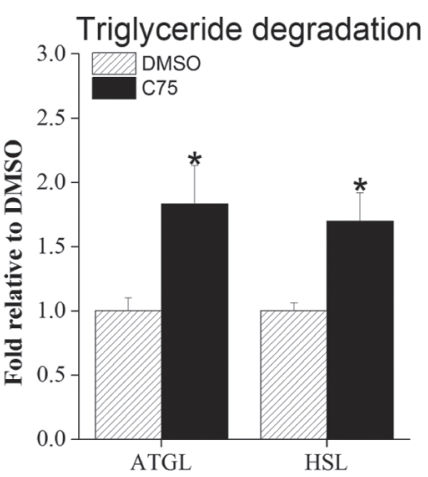

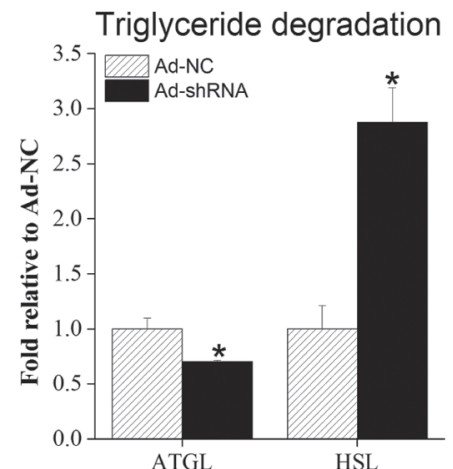

Lipid droplets forming and secretion

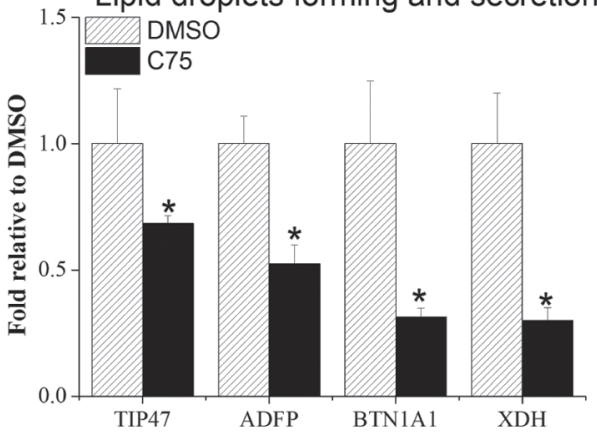

Lipid droplets forming and secretion

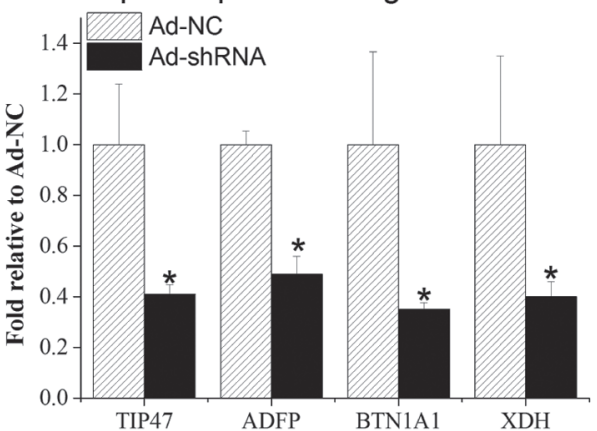

Figure 1. Effects of suppressing FA synthase (FASN) activity on the mRNA abundance of genes related to triglyceride accumulation and secretion. Goat mammary epithelial cells (GMEC) were incubated with $10 \mathrm{ng} / \mu \mathrm{L}$ of $\mathrm{C} 75$ or dimethyl sulfoxide (DMSO) for $48 \mathrm{~h}$ (A) or transfected with control adenovirus (Ad-NC) or adenovirus-short-hairpin RNA (Ad-shRNA) for $72 \mathrm{~h}$ (B). Then, total RNA was extracted for gene-expression determination by quantitative real-time PCR. Ad-NC = adenovirus containing negative shRNA for control; Ad-shRNA $=$ adenovirus containing shRNA targeting FASN sequence; GPAM = glycerol-3-phosphate acyltransferase; AGPAT6 = 1-acylglycerol-3-phosphate O-acyltransferase; $L P I N 1=$ lipin 1; DGAT = diacylglycerol acyltransferase; $A T G L=$ adipose triglyceride lipase; $H S L=$ hormone-sensitive lipase; TIP $47=$ tail-interacting protein 47 gene; $A D F P=$ adipose differentiation-related protein; $B T N 1 A 1=$ butyrophilin $1 \mathrm{~A} 1 ; X D H=$ xanthine oxidoreductase. The DMSO treatment was used as the control. Columns are the average of 3 repeats. Error bars indicate SD. ${ }^{*} P<0.05$

conjugated linoleic acid inhibited de novo FA synthesis and desaturation in bovine mammary cell cultures and suppressed milk-fat concentration in dairy cows (Loor and Herbein, 1998), a clear mechanism of how FASN affects milk-fat formation, especially in dairy goats, was still unknown. The present study was novel in that both $\mathrm{C} 75$ and shRNA treatment indicated that FASN inhibition at the gene-expression level suppressed the accumulation of TAG and lipid-droplet formation by reducing lipogenesis and esterification and promoting lipolysis in dairy goats.

Two main factors could contribute to the decrease in cellular TAG, including less lipid synthesis and more lipolysis. In the present study, reduced FASN activity and reduced FASN expression resulted not only in less mRNA expression of genes related to lipid synthesis but also increased expression of lipolytic genes. The impaired FASN function was not compensated by an alternate mechanism, thus, contributing to the decrease in lipid synthesis. We propose that, at least in vitro, goat mammary cells do not possess alternate mechanisms to synthesize TAG. Instead of accumulating cytosolic TAG, a counter-regulatory mechanism increases lipolysis resulting in rapid degradation of cellular TAG. The inhibitory effects of both $\mathrm{C} 75$ and RNA-interference treatments on TAG accumulation were likely enhanced by the increase in expression of $H S L$ that accompanied the inhibition of FASN (Figure 1A, 1B). The combined results were suggestive that the reduction of TAG content in the present study induced by inhibition of FASN was a combination of the suppression of lipogenesis and activation of lipolysis. However, more research is still needed for the issue of how the little lipolysis that occurs in lactating mammary tissue affects the physiological mechanism.

The DGAT are required for lipid-droplet formation during adipogenesis (Harris et al., 2011). Two DGAT enzymes are involved in TAG synthesis, DGAT1 and DGAT2. Despite being encoded from different gene families, DGAT1 and DGAT2 share similar enzyme 
A
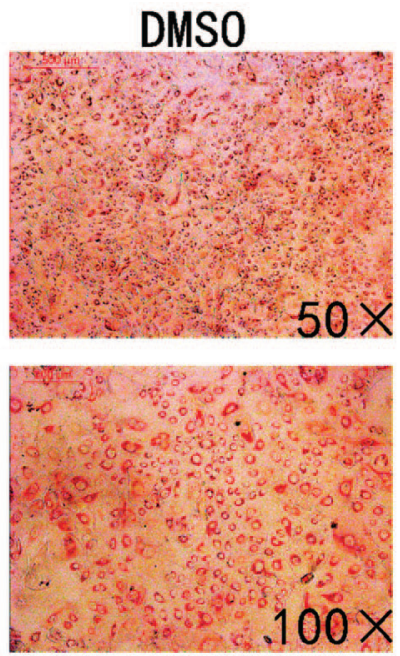

B
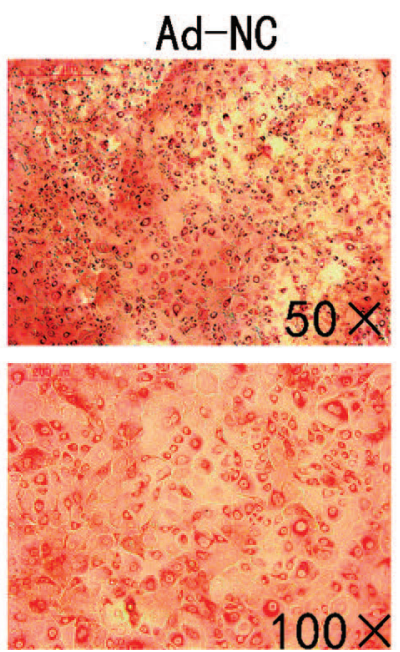

$\mathrm{C} 75$
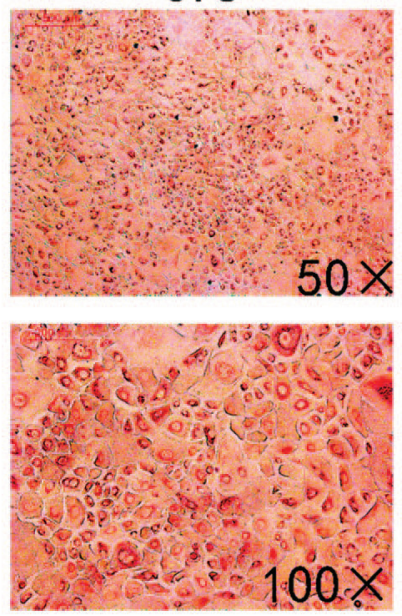

Ad-shRNA
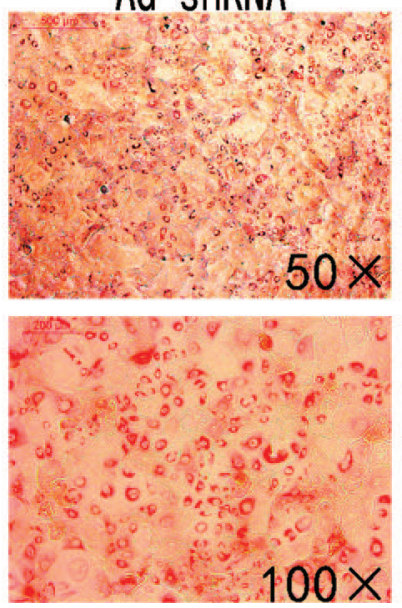
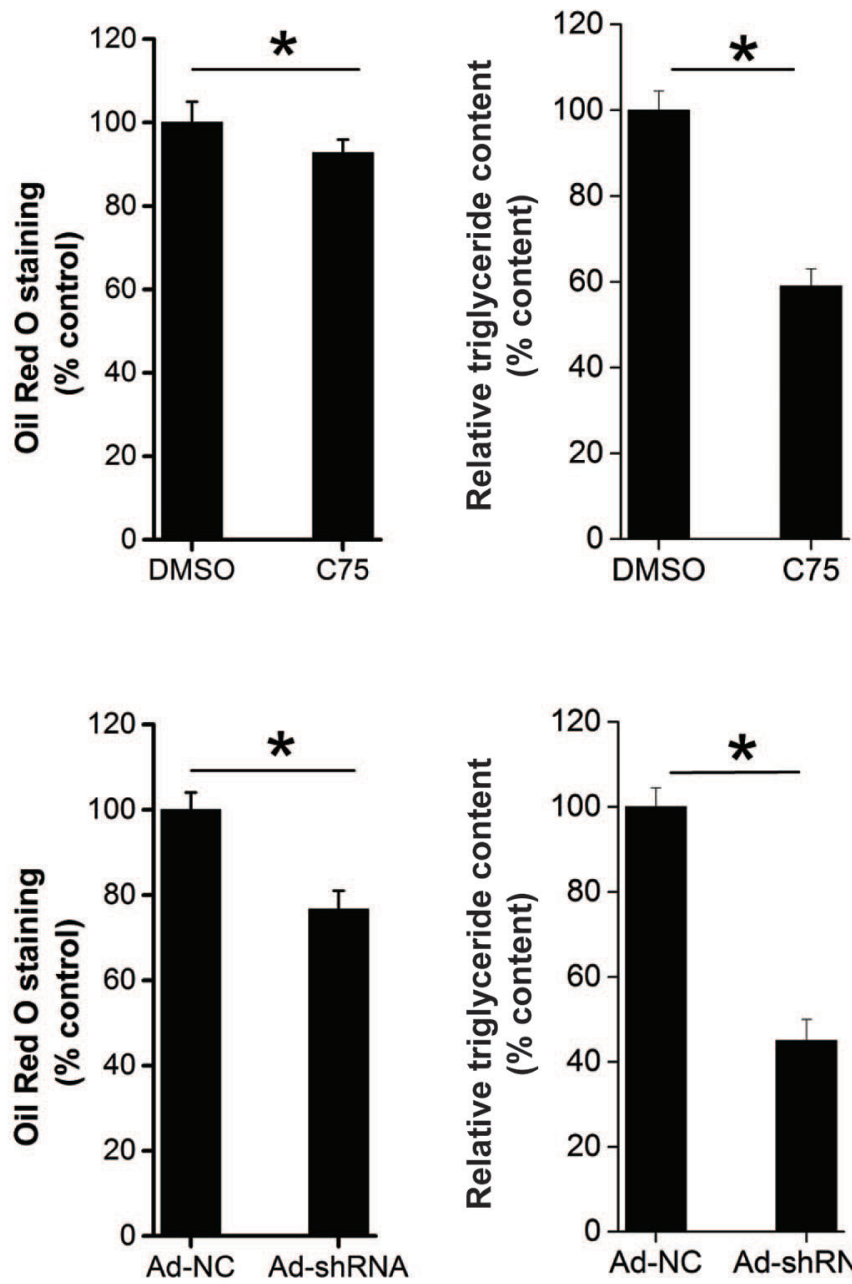

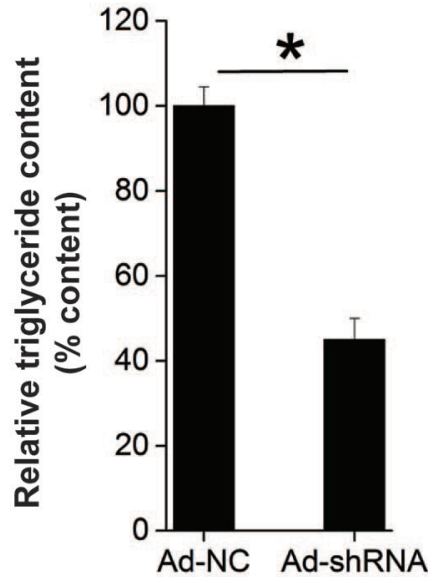

Figure 2. Effects of suppressing FA synthase (FASN) activity on cellular triglyceride content and lipid-droplets accumulation. Goat mammary epithelial cells (GMEC) were incubated with $10 \mathrm{ng} / \mu \mathrm{L}$ of C75 or dimethyl sulfoxide (DMSO) for $48 \mathrm{~h}$ (A) or transfected with control adenovirus (Ad-NC) or adenovirus-short-hairpin RNA (Ad-shRNA) for $72 \mathrm{~h}(\mathrm{~B})$. Then, the triglyceride content and lipid-droplets accumulation were determined. Ad-NC = adenovirus containing negative shRNA for control; Ad-shRNA = adenovirus containing shRNA targeting FASN sequence. Columns are the average of 6 repeats. Error bars indicate SD. ${ }^{*} P<0.05$. Color version available online.

activity and account for almost all TAG synthesis in adipocytes and the vast majority of other tissues (Ganji et al., 2004). Enzyme DGAT1 is highly expressed in the small intestine and has been suggested to be mainly involved in intestinal TAG absorption, and it accounts for $90 \%$ of intestinal DGAT activity (Cases et al., 2001; Buhman et al., 2002). In adipocytes DGAT1deficient mice shows less TAG synthesis (Smith et al., 2000). Although DGAT2 appears to compensate for the absence of DGAT1 for TAG synthesis and absorption in small intestines (Buhman et al., 2002), the highest levels of DGAT2 expression in human tissues are in liver and white adipose tissue (Cases et al., 2001). In mice, DGAT2 in liver plays an important role in hepatic TAG synthesis (Meegalla et al., 2002). The
DGAT2 (in adipose tissue) deficiency leads to severe reductions of TAG. However, the expression information and function explanatory in the mammary gland of ruminants remains unclear. In the present study, inhibition of FASN by shRNA and C75 treatment reduced the amount of cellular lipid droplets; meanwhile, the expression of DGAT2 decreased significantly by both treatments. Although decreased expression of DGAT1 was observed by silencing of FASN, the C75 treatment increased its expression (although we could not provide a logical explanation). The exact relationship between cellular lipid droplets with milk TAG synthesis is not very clear, but it could be surmised that a reduction in lipid droplets likely would result in less milk TAG for secretion. These results were similar to data from 
DGAT1 $^{-/-}$mice in which lactation was defective (Smith et al., 2000). Taken together, the data indicated that the expression of DGAT2 was responsive to FASN inhibition in goat mammary epithelial cells.

In the nonruminant mammary gland, HSL is responsible for the release of FFA from triacylglycerols, diacylglycerols, and cholesterol from the cholesteryl esters stored in the lipid droplet, to provide lipids for milk synthesis and secretion by the epithelial cells (Yeaman, 2004; Martín-Hidalgo et al., 2005). In the present study, FASN inhibition induced higher expression of $H S L$. Although prolactin was supplemented in the media, something specific to cell culture and an inability to export lipids may also have induced lipolysis partly as a self-protecting mechanism. Clearly, more research about the regulatory mechanism underlying the effect of FASN inhibition on mRNA expression of $H S L$, and the identification of the ability for lipid secretion of GMEC under the culture condition, is needed.

Consistent with the decrease of the expression of genes related to TAG synthesis, both $\mathrm{C} 75$ treatment and shRNA decreased $(P<0.05)$ the expression of TIP 47, ADFP, XDH, and BTN1A1. The PAT-family genes (perilipin, $A D F P$, and TIP 47 ) are closely linked with the synthesis of lipid droplets. The PAT proteins have a high degree of sequence similarity (Wolins et al., 2005). Essential for the formation of new lipid droplets is TIP47 (Wolins et al., 2005). For maturation of lipid droplets during milk secretion, ADFP is essential (Chong et al., 2011). Overexpression of ADFP led to a bigger size of lipid droplets and accumulation of lipid in adipocytes (Listenberger et al., 2007).

The marked reduction of TAG content in the present study is in agreement with previous studies in ADFPdeficient mice (Chang et al., 2006) and rat McA-RH7777 cells (Magnusson et al., 2006). In addition, ADFP also was reported to be crucial for milk lipid-droplet secretion by forming complexes with XDH and BTN1A1 (Chong et al., 2011). Ablation of BTN1A1 and XDH in mice resulted in impairment of cellular lipid-droplet envelopment and secretion (Ogg et al., 2004). Taken together, although a future direct assay for lipid-droplet secretion is still needed, at least in vitro, FASN seems essential for the cellular TAG accumulation and also for the expression of genes association with lipid synthesis through indirect, hence, secondary effects in goat mammary epithelial cells.

We recognize the general limitation of mRNA data in the current study. For the silencing-efficiency evaluation of FASN, the results of Western blot and quantitative RT-PCR revealed a similar silencing efficiency at the mRNA and protein level. Considering most of the genes involved in the present study were terminal en- zymes, it was likely that their mRNA expression would be positively correlated with their protein expression. Clearly, protein expression analyses will be required in the future once suitable antibodies are obtained.

Despite of the effect of FASN inhibition on gene expression and lipid metabolism in the present study, the exact mechanism underlying the effect remains unclear. As a terminal enzyme for de novo FA synthesis, previous studies indicated that FASN could help control lipid metabolism through indirect, hence, secondary effects. For instance, FASN could be involved in the control of FA synthesis and oxidation through a direct effect on the concentration of malonyl-CoA, which was shown to help control lipid metabolism through inhibition (allosteric) of $\beta$-oxidation by carnitine palmitoyltransferase 1 (CPT1; Saggerson, 2008; Foster, 2012). In addition, FASN also helps generate ligands for transcription regulators, including peroxisome proliferator-activated receptors (PPAR; Chakravarthy et al., 2009, Georgiadi and Kersten, 2012), sterol-regulatory element binding protein 1 (SREBP1), hepatocyte nuclear factor 4a (HNF4 $\alpha$ ), NF-E2-related factor-2 (NRF2), and toll-like receptor 4 (TLR4; Georgiadi and Kersten, 2012), all of which are important for lipid metabolism regulation. Fatty acid synthase also may affect protein activity indirectly, e.g., endothelial nitric-oxide synthase, through palmitoylation (Wei et al., 2011). Last, FASN may interact directly with caveolin-1 and lipid raft, which was involved in lipid secretion (Di Vizio et al., 2008). However, the exact mechanism of how FASN affects the expression of genes related to milk-fat synthesis remains unclear.

In conclusion, the inhibition of FASN can induce a widespread suppression of triglyceride accumulation and the expression of genes related to TAG synthesis and lipid-droplets formation and secretion. Understanding the regulatory role of FASN in lipid metabolism in the lactating ruminant may contribute to the efforts in controlling the milk-fat formation.

\section{ACKNOWLEDGMENTS}

This research was jointly supported by the National Natural Science Foundation of China (Beijing, China; 31072013 and 31372281), the "Transgenic New Species Breeding Program of China (2014ZX08009-051B)," and the Special Fund for Agro-Scientific Research in the Public Interest (China; 201103038).

\section{REFERENCES}

Bernard, L., J. Rouel, C. Leroux, A. Ferlay, Y. Faulconnier, P. Legrand, and Y. Chilliard. 2005. Mammary lipid metabolism and 
milk fatty acid secretion in Alpine goats fed vegetable lipids. J. Dairy Sci. 88:1478-1489.

Buhman, K. K., S. J. Smith, S. J. Stone, J. J. Repa, J. S. Wong, F. F. Knapp, B. J. Burri, R. L. Hamilton, N. A. Abumrad, and R. V. Farese. 2002. DGAT1 is not essential for intestinal triacylglycerol absorption or chylomicron synthesis. J. Biol. Chem. 277:25474-25479.

Cases, S., S. J. Stone, P. Zhou, E. Yen, B. Tow, K. D. Lardizabal, T. Voelker, and R. V. Farese Jr. 2001. Cloning of DGAT2, a second mammalian diacylglycerol acyltransferase, and related family members. J. Biol. Chem. 276:38870-38876.

Chakravarthy, M. V., I. J. Lodhi, L. Yin, R. R. Malapaka, H. E. Xu, J. Turk, and C. F. Semenkovich. 2009. Identification of a physiologically relevant endogenous ligand for PPARalpha in liver. Cell 138:476-488.

Chang, B. H., L. Li, A. Paul, S. Taniguchi, V. Nannegari, W. C. Heird, and L. Chan. 2006. Protection against fatty liver but normal adipogenesis in mice lacking adipose differentiation-related protein. Mol. Cell. Biol. 26:1063-1076.

Chirala, S. S., A. Jayakumar, Z. W. Gu, and S. J. Wakil. 2001. Human fatty acid synthase: Role of interdomain in the formation of catalytically active synthase dimer. Proc. Natl. Acad. Sci. USA 98:3104-3108

Chong, B. M., P. Reigan, K. D. Mayle-Combs, D. J. Orlicky, and J. L. McManaman. 2011. Determinants of adipophilin function in milk lipid formation and secretion. Trends Endocrinol. Metab. $22: 211-217$.

Currie, E., A. Schulze, R. Zechner, T. C. Walther, and R. V. Farese Jr. 2013. Cellular fatty acid metabolism and cancer. Cell Metab. 18:153-161.

De Schrijver, E., K. Brusselmans, W. Heyns, G. Verhoeven, and J. V. Swinnen. 2003. RNA interference-mediated silencing of the fatty acid synthase gene attenuates growth and induces morphological changes and apoptosis of LNCaP prostate cancer cells. Cancer Res. 63:3799-3804.

Di Vizio, D., R. M. Adam, J. Kim, R. Kim, F. Sotgia, T. Williams, F. Demichelis, K. R. Solomon, M. Loda, M. A. Rubin, M. P. Lisanti, and M. R. Freeman. 2008. Caveolin-1 interacts with a lipid raftassociated population of fatty acid synthase. Cell Cycle 7:22572267.

Dils, R. R. 1986. Comparative aspects of milk fat synthesis. J. Dairy Sci. 69:904-910.

Foster, D. W. 2012. Malonyl-CoA: The regulator of fatty acid synthesis and oxidation. J. Clin. Invest. 122:1958-1959.

Ganji, S. H., S. Tavintharan, D. Zhu, Y. Xing, V. S. Kamanna, and M. L. Kashyap. 2004. Niacin noncompetitively inhibits DGAT2 but not DGAT1 activity in HepG2 cells. J. Lipid Res. 45:1835-1845.

Georgiadi, A., and S. Kersten. 2012. Mechanisms of gene regulation by fatty acids. Adv. Nutr. 3:127-134.

Hansen, H. O., I. Grunnet, and J. Knudsen. 1984. Triacylglycerol synthesis in goat mammary gland. Factors influencing the esterification of fatty acids synthesized de novo. Biochem. J. 220:521-527.

Harris, C. A., J. T. Haas, R. S. Streeper, S. J. Stone, M. Kumari, K. Yang, X. Han, N. Brownell, R. W. Gross, R. Zechner, and R. V. Farese Jr. 2011. DGAT enzymes are required for triacylglycerol synthesis and lipid droplets in adipocytes. J. Lipid Res. $52: 657-667$.

Harvatine, K. J., Y. R. Boisclair, and D. E. Bauman. 2009. Recent advances in the regulation of milk fat synthesis. Animal 3:40-54.

Kadegowda, A. K., M. Bionaz, L. S. Piperova, R. A. Erdman, and J. J. Loor. 2009. Peroxisome proliferator-activated receptor-gamma activation and long-chain fatty acids alter lipogenic gene networks in bovine mammary epithelial cells to various extents. J. Dairy Sci. 92:4276-4289

Kurokawa, J., S. Arai, K. Nakashima, H. Nagano, A. Nishijima, K. Miyata, R. Ose, M. Mori, N. Kubota, T. Kadowaki, Y. Oike, H. Koga, M. Febbraio, T. Iwanaga, and T. Miyazaki. 2010. Macrophage-derived AIM is endocytosed into adipocytes and decreases lipid droplets via inhibition of fatty acid synthase activity. Cell Metab. 11:479-492.
Listenberger, L. L., A. G. Ostermeyer-Fay, E. B. Goldberg, W. J. Brown, and D. A. Brown. 2007. Adipocyte differentiation-related protein reduces the lipid droplet association of adipose triglyceride lipase and slows triacylglycerol turnover. J. Lipid Res. 48:27512761

Loor, J. J., and J. H. Herbein. 1998. Exogenous conjugated linoleic acid isomers reduce bovine milk fat concentration and yield by inhibiting de novo fatty acid synthesis. J. Nutr. 128:2411-2419.

Magnusson, B., L. Asp, P. Bostrom, M. Ruiz, P. Stillemark-Billton, D. Linden, J. Boren, and S. O. Olofsson. 2006. Adipocyte differentiation-related protein promotes fatty acid storage in cytosolic triglycerides and inhibits secretion of very low-density lipoproteins. Arterioscler. Thromb. Vasc. Biol. 26:1566-1571.

Martín-Hidalgo, A., L. Huerta, N. Alvarez, G. Alegria, M. Del Val Toledo, and E. Herrera. 2005. Expression, activity, and localization of hormone-sensitive lipase in rat mammary gland during pregnancy and lactation. J. Lipid Res. 46:658-668.

Massaro, M., M. A. Carluccio, and R. De Caterina. 1999. Direct vascular antiatherogenic effects of oleic acid: A clue to the cardioprotective effects of the Mediterranean diet. Cardiologia 44:507-513.

Meegalla, R. L., J. T. Billheimer, and D. Cheng. 2002. Concerted elevation of acyl-coenzyme A:diacylglycerol acyltransferase (DGAT) activity through independent stimulation of mRNA expression of DGAT1 and DGAT2 by carbohydrate and insulin. Biochem. Biophys. Res. Commun. 298:317-323.

Menendez, J. A., A. Vazquez-Martin, F. J. Ortega, and J. M. Fernandez-Real. 2009. Fatty acid synthase: Association with insulin resistance, type 2 diabetes, and cancer. Clin. Chem. 55:425-438.

Moriyama, T., K. Kishimoto, K. Nagai, R. Urade, T. Ogawa, S. Utsumi, N. Maruyama, and M. Maebuchi. 2004. Soybean beta-conglycinin diet suppresses serum triglyceride levels in normal and genetically obese mice by induction of beta-oxidation, downregulation of fatty acid synthase, and inhibition of triglyceride absorption. Biosci. Biotechnol. Biochem. 68:352-359.

Morris, C. A., N. G. Cullen, B. C. Glass, D. L. Hyndman, T. R. Manley, S. M. Hickey, J. C. McEwan, W. S. Pitchford, C. D. Bottema, and M. A. Lee. 2007. Fatty acid synthase effects on bovine adipose fat and milk fat. Mamm. Genome 18:64-74.

Ogg, S. L., A. K. Weldon, L. Dobbie, A. J. Smith, and I. H. Mather. 2004. Expression of butyrophilin (Btn1a1) in lactating mammary gland is essential for the regulated secretion of milk-lipid droplets. Proc. Natl. Acad. Sci. USA 101:10084-10089.

Olsen, C. P. 2011. Why make fat?: The impact of de novo fatty acid synthesis on fat storage, reproduction, and longevity in C. elegans. Pages 1-148. Doctoral Dissertation. Univ. Washington.

Peterson, D. G., E. A. Matitashvili, and D. E. Bauman. 2004. The inhibitory effect of trans-10, cis-12 CLA on lipid synthesis in bovine mammary epithelial cells involves reduced proteolytic activation of the transcription factor SREBP-1. J. Nutr. 134:2523-2527.

Rendina, A. R., and D. Cheng. 2005. Characterization of the inactivation of rat fatty acid synthase by C75: Inhibition of partial reactions and protection by substrates. Biochem. J. 388:895-903.

Saggerson, D. 2008. Malonyl-CoA, a key signaling molecule in mammalian cells. Annu. Rev. Nutr. 28:253-272.

Smith, S. J., S. Cases, D. R. Jensen, H. C. Chen, E. Sande, B. Tow, D. A. Sanan, J. Raber, R. H. Eckel, and R. V. Farese Jr. 2000. Obesity resistance and multiple mechanisms of triglyceride synthesis in mice lacking Dgat. Nat. Genet. 25:87-90.

Suburu, J., L. Shi, J. Wu, S. Wang, M. Samuel, M. J. Thomas, N D. Kock, G. Yang, S. Kridel, and Y. Q. Chen. 2014. Fatty acid synthase is required for mammary gland development and milk production during lactation. Am. J. Physiol. Endocrinol. Metab. 306:E1132-1143.

Thupari, J. N., L. E. Landree, G. V. Ronnett, and F. P. Kuhajda. 2002. C75 increases peripheral energy utilization and fatty acid oxidation in diet-induced obesity. Proc. Natl. Acad. Sci. USA 99:9498-9502.

Wang, W. 2009. Establishment of goat mammary gland epithelial cell line and the study on fatty acid synthase gene by RNA interfer- 
ence. Pages 1-77. PhD Dissertation. Northwest A\&F Univ., College of Animal Science and Technology, Yangling, Shaanxi.

Wei, X., J. G. Schneider, S. M. Shenouda, A. Lee, D. A. Towler, M. V. Chakravarthy, J. A. Vita, and C. F. Semenkovich. 2011. De novo lipogenesis maintains vascular homeostasis through endothelial nitric-oxide synthase (eNOS) palmitoylation. J. Biol. Chem. $286: 2933-2945$.

Wolins, N. E., B. K. Quaynor, J. R. Skinner, M. J. Schoenfish, A. Tzekov, and P. E. Bickel. 2005. S3-12, Adipophilin, and TIP47 package lipid in adipocytes. J. Biol. Chem. 280:19146-19155.

Yeaman, S. J. 2004. Hormone-sensitive lipase-New roles for an old enzyme. Biochem. J. 379:11-22.
Yonezawa, T., S. Haga, Y. Kobayashi, K. Katoh, and Y. Obara. 2008. Unsaturated fatty acids promote proliferation via ERK1/2 and Akt pathway in bovine mammary epithelial cells. Biochem. Biophys. Res. Commun. 367:729-735.

Yonezawa, T., S. Haga, Y. Kobayashi, K. Katoh, and Y. Obara. 2009. Short-chain fatty acid signaling pathways in bovine mammary epithelial cells. Regul. Pept. 153:30-36.

Zhu, J. J., J. Luo, W. Wang, K. Yu, H. B. Wang, H. B. Shi, Y. T. Sun, X. Z. Lin, and J. Li. 2014. Inhibition of FASN reduces the synthesis of medium-chain fatty acids in goat mammary gland. Animal 8:1469-1478. 